\title{
The Impact of Assimilating Atmospheric Infrared Sounder Observation on the Forecast of Typhoon Tracks
}

\author{
Chien-Ben Chou $^{1}$ and Huei-Ping Huang ${ }^{2}$ \\ ${ }^{1}$ Meteorological Satellite Center, Central Weather Bureau, Taipei 10048, Taiwan \\ ${ }^{2}$ School for Engineering of Matter, Transport, and Energy, Arizona State University, Tempe, AZ 85281, USA
}

Correspondence should be addressed to Huei-Ping Huang, hp.huang@asu.edu

Received 19 July 2011; Revised 3 November 2011; Accepted 30 November 2011

Academic Editor: Zhanqing Li

Copyright (C) 2011 C.-B. Chou and H.-P. Huang. This is an open access article distributed under the Creative Commons Attribution License, which permits unrestricted use, distribution, and reproduction in any medium, provided the original work is properly cited.

\begin{abstract}
This work assesses the effects of assimilating atmospheric infrared sounder (AIRS) observations on typhoon prediction using the three-dimensional variational data assimilation (3DVAR) and forecasting system of the weather research and forecasting (WRF) model. Two major parameters in the data assimilation scheme, the spatial decorrelation scale and the magnitude of the covariance matrix of the background error, are varied in forecast experiments for the track of typhoon Sinlaku over the Western Pacific. The results show that within a wide parameter range, the inclusion of the AIRS observation improves the prediction. Outside this range, notably when the decorrelation scale of the background error is set to a large value, forcing the assimilation of AIRS data leads to degradation of the forecast. This illustrates how the impact of satellite data on the forecast depends on the adjustable parameters for data assimilation. The parameter-sweeping framework is potentially useful for improving operational typhoon prediction.
\end{abstract}

\section{Introduction}

The atmospheric infrared sounder (AIRS) is a state-of-theart hyperspectral infrared sensor that has provided critical observational data for weather and climate analysis since its launch in 2002 (McNally et al. [1], Chahine et al. [2]). The AIRS spectrum consists of 2378 channels from $3.7 \mu \mathrm{m}-$ $15.4 \mu \mathrm{m}$ with a spectral resolution of $\Delta \lambda / \lambda=1 / 1200$. The cross-track swath width is $1650 \mathrm{~km}$ and spatial resolution is $13.5 \mathrm{~km}$ at the nadir field of view (Aumann et al. [3], Chahine et al. [2]). Due to its high resolution and accuracy (errors are within $1 \mathrm{~K}$ for the temperature of a $1 \mathrm{~km}$ vertical layer and $20 \%$ for the lower tropospheric moisture of a $2 \mathrm{~km}$ vertical layer, http://airs.jpl.nasa.gov/), AIRS can potentially provide high-quality temperature and humidity data for applications that would otherwise rely on conventional sounding. Given its uniformly high spatial resolution, the strategy for assimilating the AIRS retrieval data into a weather forecast system may differ from that for assimilating conventional soundings. With the increasing usage of AIRS retrieval products in data assimilation for regional weather prediction (e.g., [4$7]$ ), it remains an outstanding problem to determine the ideal weight given to the AIRS retrieval profiles (and satellite observation in general) in order to optimize its impact on the forecast. As a contribution to this topic, this work will first examine the usefulness of assimilating the AIRS retrieval data for the prediction of typhoon tracks then explore the sensitivity of the forecast error on the adjustable parameters (that determine the weight given to the satellite observation) in the data assimilation procedure.

We choose to investigate the forecast of typhoon track because it exemplifies the situation when conventional sounding does not provide sufficient information for model initialization (see a survey in Barker et al. [8]). Recently, attempts have been made to assimilate high-resolution satellite observations of temperature (and the derived velocity) and moisture fields into a regional model for typhoon or hurricane predictions (e.g., Zhu et al. [9], Zhang et al. [10], Chou et al. [11], Goerss [12], Li and Liu [5]). Not often discussed is how the forecast outcome depends on the adjustable parameters in the data assimilation procedure. For example, when assimilating sparsely distributed sounding data with large "gaps" over the ocean, the introduction of background error with a large spatial decorrelation scale 


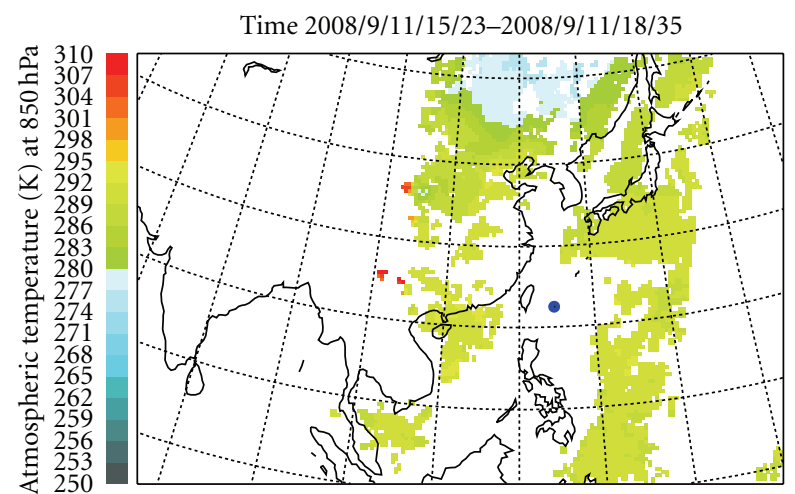

(a)

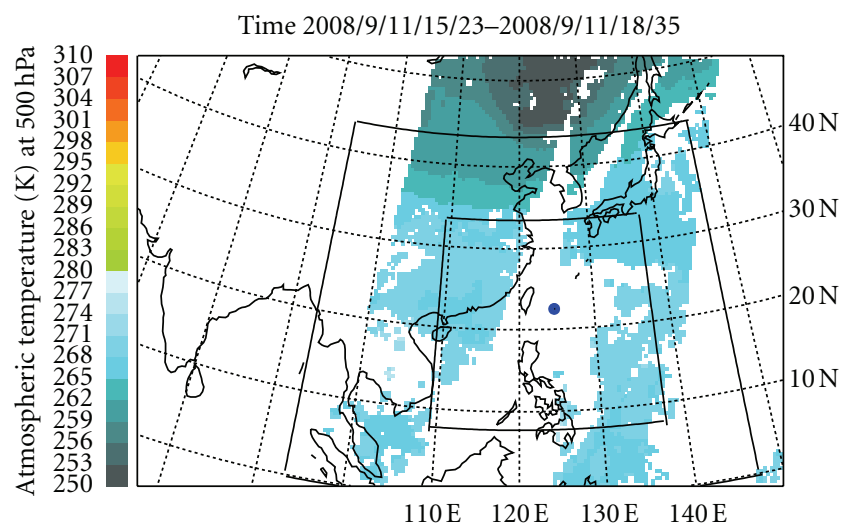

(b)

FIgURE 1: (a) The $850 \mathrm{hPa}$ retrieval temperature from AIRS observation within the 3 hours time window for data assimilation for the runs initialized at 1800 UTC, 11 September 2008. Blue filled circle is the center of typhoon. (b) is same as (a) but for temperature at $500 \mathrm{hPa}$. The nested domains for WRF model simulations are superimposed in panel (b).

helps constrain the procedure such that a harmful smallscale irregularity introduced by a poor observation will be smoothed over. If the same constraint is applied to the assimilation of satellite observations, it may instead act to erase useful fine-scale information in the satellite data. We will test this point in this study. For a useful sensitivity test, we will adopt a parameter-sweeping strategy to perform multiple forecast runs by varying the decorrelation length scale and the variance of the background error, thereby implicitly varying the weight given to the satellite observation at different scales.

\section{Data and Methodology}

2.1. Model and 3DVAR System. We will use the weather research and forecasting (WRF) model Version 3 (Skamarock et al. [13]) to perform data assimilation and the forecast of typhoon tracks. The model domains are nested (see Figure 1(b)). The outer domain covers the Western Pacific and East Asia with $135 \times 120$ (west-east $\times$ south-north) grid points and $45 \mathrm{~km}$ horizontal resolution. The inner domain has $210 \times 210$ grid points with $15 \mathrm{~km}$ resolution. The vertical resolution is fixed at 23 levels.
For data assimilation, we use WRF's 3DVAR system, which is ideal for executing our sensitivity experiments. The description of the basic procedure in that system can be found in Barker et al. [8] and WRF-ARW Version 3 Users Guide [14]. The theory and implementation for variational data assimilation in numerical weather prediction are well established (e.g., Lorenc [15]). Therein, we assume that the probability density function of the background error and observation error are Gaussian with no bias and the two are independent of each other. The optimal atmospheric state, $x$, can be found as the one that minimizes the cost function:

$$
\begin{aligned}
J(x)= & \left(x-x^{b}\right)^{T} \mathbf{B}^{-1}\left(x-x^{b}\right)+\left\{y(x)-y^{0}\right\}^{T} \\
& \times(\mathbf{E}+\mathbf{F})^{-1}\left\{y(x)-y^{0}\right\},
\end{aligned}
$$

where $x$ is the analysis state, $x^{b}$ is the background state, $\mathbf{B}$ is the background error covariance matrix, $y$ is the forward projection of the analysis state onto the observation space, $y^{0}$ is observation, $\mathbf{E}$ is the observation error covariance matrix, and $\mathbf{F}$ is the representativity error covariance matrix.

In our study, $y^{0}$ contains the temperature and moisture profiles retrieved from AIRS, based on NASA's archive (see Section 2.2). The background error covariance matrix is taken from the CV3 default (but then perturb it, see below) of WRF 3DVAR system, which was generated by the NMC method (Parrish and Derber [16]) based on the difference between the 24-hr and 48-hr forecast, using a global model with T170 resolution (WRF-ARW Version 3 User's Guide [14, Chapter 6]). Although there exists the CV5 option that uses a regional model to generate the background covariance matrix, we choose the simpler CV3 since its default values for the covariance matrix are uniquely defined and are immediately available to all WRF users (whereas CV5 depends on the detailed setup of the regional model by individual users). The global model (NCEP GFS) has an effective spatial resolution of about $100 \mathrm{~km}$ in the subtropics, coarser than the resolution used in our simulations for typhoons. By starting with the default setting, our study serves yet another purpose of testing the sensitivity of the outcome of WRF's predictions when we perturb the background error covariance away from the default. The perturbation will be done in a series of sensitivity experiments by adjusting the spatial decorrelation scale and the amplitude of the background error covariance, as detailed in Section 2.3. The scope of our investigation is less ambitious than recent theoretical explorations (e.g., Desroziers and Ivanov [17], Sadiki and Fischer [18]) on systematic ways to adjust the B matrix a posteriori. Nevertheless, the practical examples shown in this paper should provide a useful guidance for future work that aims to make progress in that direction but for the complicated task of typhoon prediction.

2.2. Satellite Data. Although the incorporation of satellite data in typhoon or hurricane prediction has become more common, the usage of AIRS retrieval data in those predictions is still new. A few recent studies have demonstrated potential improvements in typhoon/hurricane prediction when AIRS observations were incorporated into the WRF 
data assimilation system [5-7]. The AIRS observations we use are the retrieved temperature and moisture profiles from the NASA archive of the along-track data, available at http://airs.jpl.nasa.gov/. The detail of the dataset and the relevant retrieval procedures can be found in Susskind et al. [19-21], and Olsen et al. [22]. The retrieval of temperature and moisture profiles were based on a statistical algorithm that did not involve the use of an atmospheric GCM, except for the forecasted surface pressure which was used as the lower boundary condition for calculating the expected radiance for a given meteorological state. Given such, in our experiments, it may be reasonable to assume that the observation error of AIRS data and the background error are independent in the variational procedure. The AIRS dataset was constructed with applications of data assimilation in mind; all products in the AIRS archive come with error estimates, which we use as a guidance of setting the observation errors in the variational procedure (see below).

In addition to AIRS observations, the Advanced Microwave Sounding Unit (AMSU) observations were also used in the retrieval procedure to create the archived AIRS profiles used in this work. (We will call the dataset "AIRS" for brevity.) The AIRS data has a higher horizontal resolution (13.5 $\mathrm{km}$ at nadir) compared to AMSU (40 km at nadir). In the retrieval procedure, 9 fields of view of AIRS are collocated and embedded in one AMSU field of view (Susskind et al. $[19,20])$ to create a single profile of temperature and moisture. The combined retrieval products have a horizontal resolution similar to that of AMSU, at $\sim 40 \mathrm{~km}$. The archived profiles have 28 vertical levels (similar to the standard pressure levels in conventional observations) from $1000 \mathrm{hPa}$ to $0.1 \mathrm{hPa}$ for temperature, and 15 levels from $1000 \mathrm{hPa}$ to $50 \mathrm{hPa}$ for humidity. The vertical resolution is $100 \mathrm{hPa}$ in midtroposphere and higher near the surface and tropopause. The data within the troposphere are used for assimilation.

In our experiment, we do not assimilate all AIRS retrieval data in the model domain. Instead, we retain only the "Level 2" retrieval data that passed NASA's quality control. For the retrieved temperature, they are the profiles with an estimated error of less than $1^{\circ} \mathrm{K}$. This prescreening helps eliminate the retrieval profiles that have large errors at near the tropopause or the surface. Given that the $1^{\circ} \mathrm{K}$ error in temperature is comparable to the default error estimate for conventional sounding in the WRF 3DVR system (detailed in the file, /WRFDA/var/obsproc/TEMP.txt in WRF package), we then use the default sounding error in the WRF 3DVAR system as their observation error. A communication with NASA AIRS Team Susskind et al. [21], personal communication) affirmed that this is a reasonable approach.

The quality control eliminates many satellite profiles located in the vicinity of the core of the typhoon, as shown in Figure 1. However, the retained satellite data still cover large areas over the outflow regions of the typhoon. Given that the quality-checked satellite data has more influence on the outflow than the inner core structure, this study will focus on the impact of satellite observation on the prediction of typhoon tracks, which is affected by the interaction of the outflow and the large-scale environment. For the forecast made without the input of the AIRS data, the NCEP global forecast is directly interpolated onto the regional WRF grids without further assimilation of other data. For the runs that incorporate the AIRS data, we use the NCEP global forecast as the first guess for the WRF 3DVAR system to assimilate the AIRS retrieval profiles.

Previous studies have discussed "data thinning" (e.g., Liu and Rabier [23], Bauer et al. [24]) in variational data assimilation as a way to conform to the assumption that the observation error matrix is diagonal, that is, it contains no spatial correlation (and no correlation between two channels for satellite data). While there were examples that showed the benefit of data thinning (e.g., Liu and Rabier [23]), there have also been concerns that useful information can be lost if the thinning is overdone (e.g., Bauer et al. [24]). For satellite data, recent studies (Bormann and Bauer [25], Bormann et al. [26]) indicate that the spatial and interchannel correlation of the error in radiance is weak for the temperature channels in AMSU and for the longwavelength part of AIRS. The correlation is somewhat stronger for water vapor channels, and for short-wavelength part of AIRS temperature channels. These recent studies suggested that the spatial and interchannel correlation in the AIRS/AMSU data might not be as strong as previously thought. While we use the retrieval product instead of the radiance for assimilation, the weak correlation in radiance might translate at least in part to a weak correlation in the retrieved temperature profiles, given that the AIRS retrieval procedure does not involve running an atmospheric model (which could lead to additional spatial correlation) except for adopting the predicted surface pressure for defining the lower boundary condition for calculating the expected radiance at a location. Moreover, since we use the combined AIRSAMSU retrieval product with an effective resolution closer to AMSU ( $\sim 40 \mathrm{~km})$, the spatial correlation may not be as strong as the case when only AIRS ( $\sim 13 \mathrm{~km}$ resolution) is used. With these considerations, we adopt a simpler approach without data thinning. We communicated with NASA AIRS Team on the treatment of observation error and received an affirmation ([21], personal communication) that recent data assimilation experiments carried out by members of AIRS Team also assumed that the errors of the retrieved AIRS temperature profiles are uncorrelated.

2.3. Sensitivity Experiments. While sensitivity experiments with varied background errors have not been systematically carried out for the assimilation of satellite data in typhoon prediction, a few studies have examined the impact of tuning the background error on the prediction of typhoon using conventional observations ( $\mathrm{Gu}$ et al. [27], Guo et al. [28]). Following the practice in those studies, we will change the background error in WRF's data assimilation procedure by adjusting the parameters LEN_SCALING15 (five parameters for the decorrelation length scale of the background error for the perturbation streamfunction, velocity potential, temperature, humidity, and surface pressure) and VAR_SCALING1-5 (the variance of background error for the same five variables). Specifically, we will vary both parameters over the range of 0.1-1.0 (1.0 being the default value for WRF). How a specific parameter value 


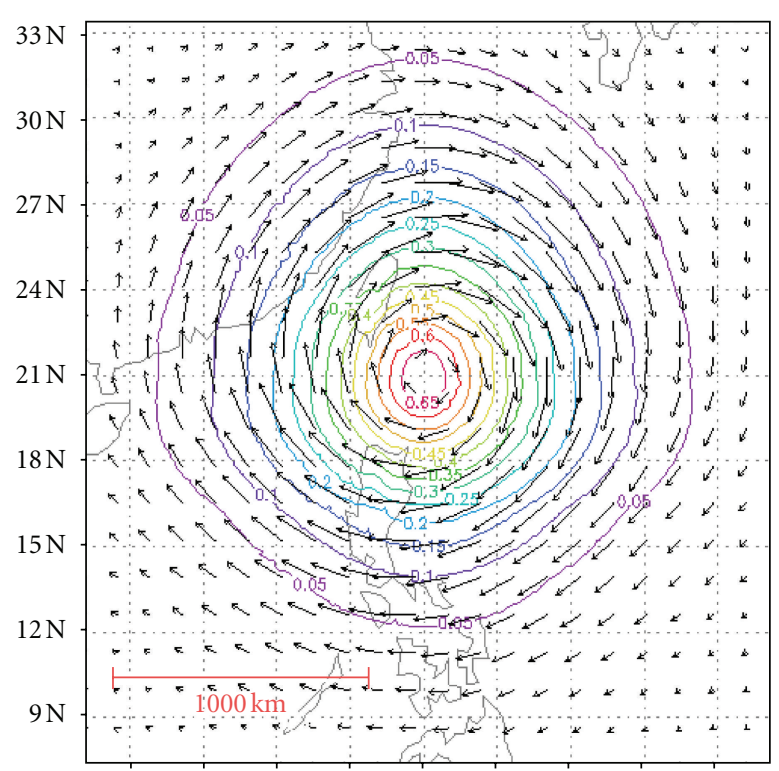

$111 \mathrm{E} 114 \mathrm{E} 117 \mathrm{E} \quad 120 \mathrm{E} 123 \mathrm{E} \quad 126 \mathrm{E} 129 \mathrm{E} 132 \mathrm{E} 135 \mathrm{E}$

$\overrightarrow{1}$

(a)

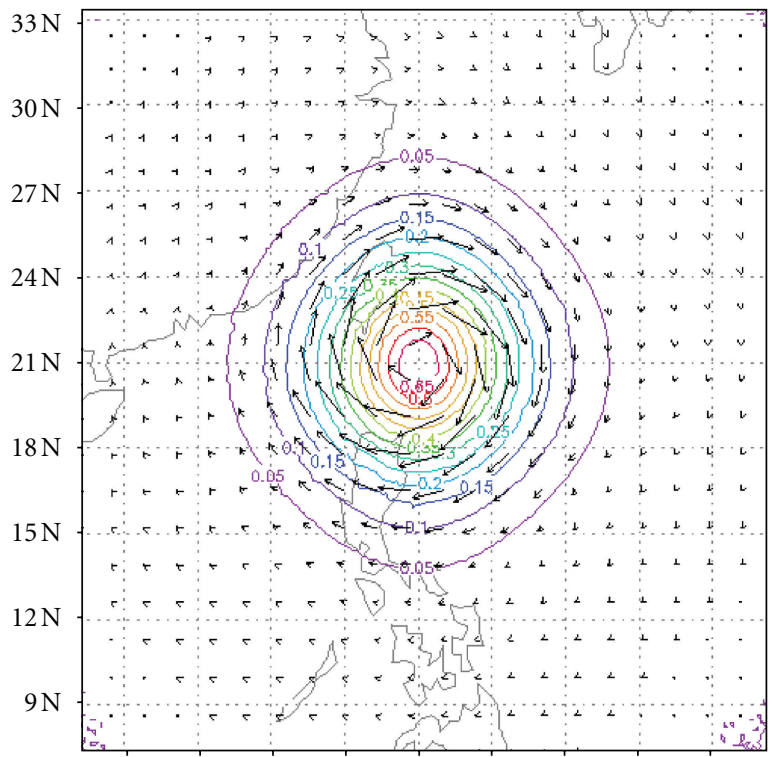

111E 114E 117E $120 \mathrm{E} 123 \mathrm{E} \quad 126 \mathrm{E} \quad 129 \mathrm{E} \quad 132 \mathrm{E} \quad 135 \mathrm{E}$ $\overrightarrow{2}$

(b)

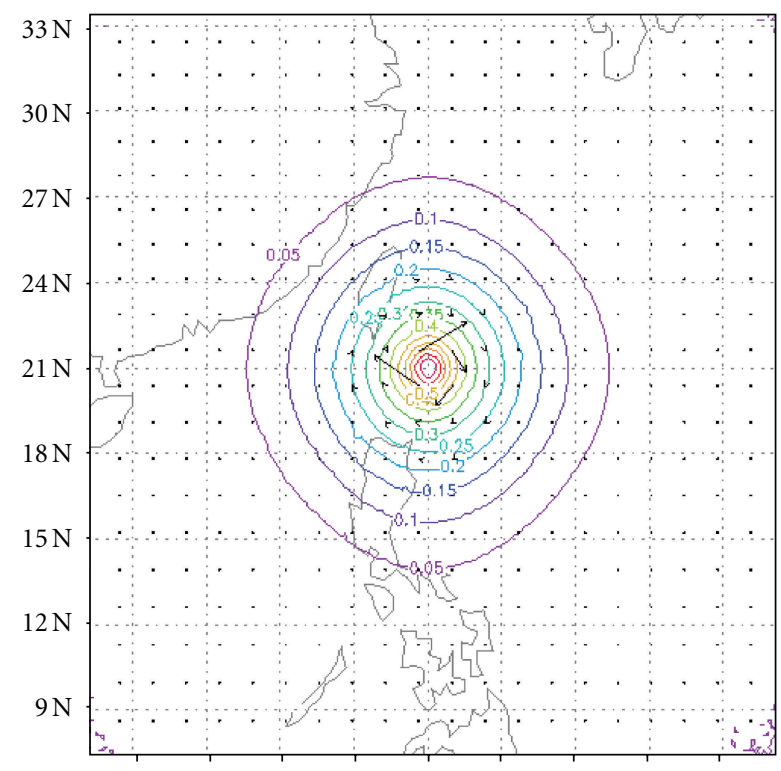

111E 114E 117E 120E 123E 126E 129E 132E 135E

$\overrightarrow{6}$

(c)

FIGURE 2: The incremental adjustment in temperature (contours) and velocity (vectors) at $500 \mathrm{hPa}$ as a result of a "single observation" experiment for WRF 3DVAR with a $1^{\circ} \mathrm{K}$ temperature perturbation given at a grid point. The three panels are with (a) LEN_SCALING = 1.0 , (b) LEN_SCALING $=0.5$, and (c) LEN_SCALING $=0.1$. All three cases are with VAR_SCALING $=1.0$. The scale of velocity vector is indicated below each panel. The red bar in panel (a) marks the distance of $1000 \mathrm{~km}$.

of LEN_SCALING corresponds to a physical length scale depends on the resolution and the general settings (e.g., model domain and latitude) of the model. In midlatitude, the decorrelation scale is generally of $O(1000 \mathrm{~km})$ based on a "single-observation" test if LEN_SCALING is set to 1.0, for example, Rizvi [29]. To verify the physical length scale corresponding to different values of LEN_SCALING in our experiments, we performed single-observation tests by running WRF 3DVAR with an imposed $1^{\circ} \mathrm{K}$ perturbation in temperature at a single grid point located southeast of Taiwan. Figure 2 shows the analysis increment in $500 \mathrm{hPa}$ temperature and velocity induced by the single observation for LEN_SCALING $=1.0,0.5$, and 0.1 , all with VAR_SCALING $=1.0$. It is found that the spatial decorrelation scale decreases from over $500 \mathrm{~km}$ for LEN_SCALING = 1.0 to about $200 \mathrm{~km}$ for LEN_SCALING $=0.1$. We have also 
performed similar experiments by changing VAR_SCALING to 0.5 and 0.1 (not shown). As expected, VAR_SCALING only affected the amplitude (which decreases with a reduced VAR_SCALING), but not the spatial scale, of the response. In our experiments, we are not overly concerned about targeting a specific length scale, but rather ensuring that the sensitivity experiments are performed over a wide enough range in the parameter space to reveal the dependence of the forecast error on the relevant parameters. Our ensuing discussion will only refer to the parameter values of LEN_SCALING1-5 and VAR_SCALING1-5. Moreover, to simplify the experiment, the length scale or variance for the five variables will be adjusted to the same value (e.g., if LEN_SCALING1 is set to 0.5 , so are all LEN_SCALING2-5). We will refer the two groups of parameters as "LEN_SCALING" and "VAR_SCALING."

\section{Forecast of Typhoon Tracks}

We choose Typhoon Sinlaku (2008) for the forecast experiments. It first developed over the Western Pacific and moved to the east of Philippines on 9 September 2008. It landed in northern Taiwan at 0150 UTC on 14 September 2008 and eventually left the island at 1000 UTC on the same day. Afterward, it slowly moved northwestward, turning northward to Japan at 0800 UTC on 15 September 2008. Our primary simulations will be for the period from 1800 UTC, 11 September 2008 (when the model is initialized), to 1800 UTC, 15 September 2008, which covers the major events of its landing and the changes in the direction of its track. (In this case, the initial field is from an 18-hr forecast.) At the chosen initial time, the center of the typhoon was located approximately $300 \mathrm{~km}$ east of Taiwan. This initial time is chosen because AIRS observations are available for the relevant region within the 3-hour window for data assimilation: see Figure 1 for the relevant AIRS swath which covers most of the WRF model domain used for the forecast. The usable (quality-controlled) temperature/moisture profiles from AIRS (colored regions in Figure 1) fill sufficiently large areas of the typhoon outflow region such that we expect an impact of the satellite data on the forecast.

3.1. Primary Forecast Experiments. In the primary set of experiments, we choose three values of the parameter LEN_SCALING (the decorrelation length scale for the background error) as 1.0, 0.5, and 0.1 (1.0 is the default value in the WRF 3DVAR system). For each chosen value of LEN_SCALING, the second parameter VAR_SCALING is varied from 0.1 to 1.0 step 0.1 . This consists of 3 sets of 10 forecast runs with 30 different combinations of (LEN_SCALING, VAR_SCALING). Figure 3 summarizes the predicted typhoon tracks and the corresponding forecast error (measured by the difference between the predicted and observed tracks) as a function of forecast time. The control run, that is, the forecast without the assimilation of AIRS data, is shown as the black curve and the observed best track is shown as the blue curve in each panel of Figure 3. The track of typhoon Sinlaku predicted by the control run is biased toward the east such that the typhoon in the model never lands in Taiwan as it should.

When the AIRS observation is assimilated in the forecast, we find that for a fixed value of LEN_SCALING, the predicted typhoon track systematically shifts westward with an increasing VAR_SCALING. Recall that when VAR_SCALING is larger, the impact of the satellite observation is generally stronger. While we would have desired a westward shift of the predicted track to improve the forecast, when LEN_SCALING $=1.0$ (Figures 3(a) and 3(b)) and VAR_SCALING is large, the predicted tracks shifted too far west so as to produce an error that is even larger in its magnitude than the control run. On the other hand, with the smallest value of VAR_SCALING $(=0.1)$, the forecast becomes better than the control run.

When LEN_SCALING is reduced to 0.5 (Figures $3(\mathrm{c}$ ) and $3(\mathrm{~d})$ ), assimilating the AIRS observation also leads to a westward shift of the predicted tracks. The shift becomes more pronounced (but not as exaggerated as the case with LEN_SCALING $=1.0$ ) with an increasing VAR_SCALING, which was varied from 0.1 to 1.0 in Figures 3(c) and 3(d). The cases with small values (0.1 and 0.2) of VAR_SCALING produced a smaller error than the control run. Although from Figure 3(c) the predicted tracks under most values of VAR_SCALING appear to be closer to the observed best track compared to the control run, the error chart in Figure 3(d) indicates that the cases with VAR_SCALING > 0.3 have a larger error than the control run. This comes mainly from the error in the speed of movement of the typhoon. When LEN_SCALING is further reduced to 0.1 (under which VAR_SCALING is varied from 0.1 to 1.0 as before; Figures 3(e) and 3(f)), the forecast still shows the tendency of a westward shift of the track with increasing VAR_SCALING. For most values of VAR_SCALING from $0.1-1.0$, the assimilation of AIRS data led to a reduced forecast error for the track compared to the control run.

3.2. Discussion and Further Experiments. The results from these experiments demonstrate the potential of using the AIRS data to improve typhoon prediction. They also reveal a wide range of behavior of the forecast when the key parameters, LEN_SCALING and VAR_SCALING, for the background error are tuned in the data assimilation procedure. This underscores the importance of tuning these parameters for specific applications.

In Figure 3, the better cases of forecast are those with a small to moderate (still smaller than the default) value of LEN_SCALING and a moderate value of VAR_SCALING. The dependence on LEN_SCALING is more clear. The reason that a smaller value of LEN_SCALING produced a better prediction is likely because it allows the system to retain useful small-scale features in the AIRS observation, which would otherwise be suppressed when LEN_SCALING is large. Recall that the default background error covariance in the WRF 3DVAR system was constructed using a global model with a relatively coarse $(\sim 100 \mathrm{~km})$ horizontal resolution. The default setting might have been more ideal for assimilating conventional, coarse-resolution soundings. The AIRS data 


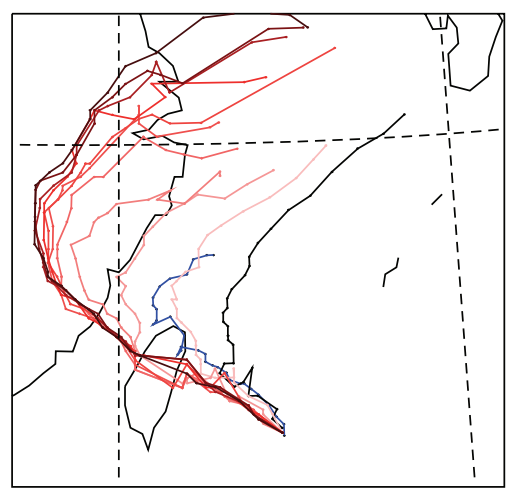

(a)

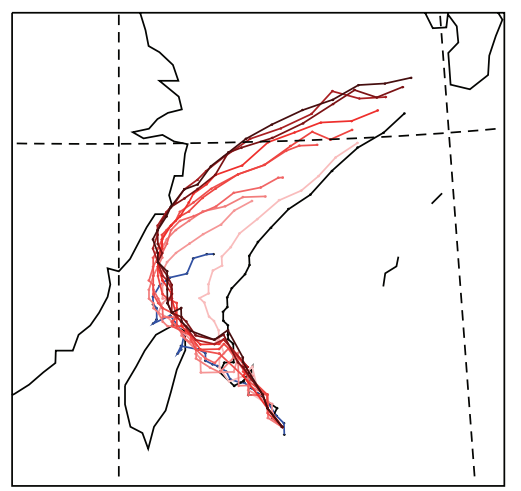

(c)

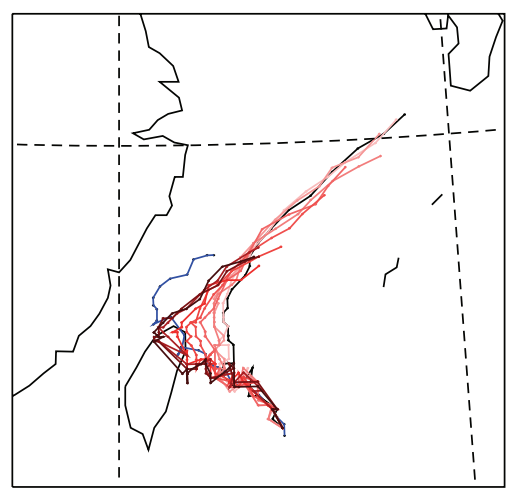

(e)

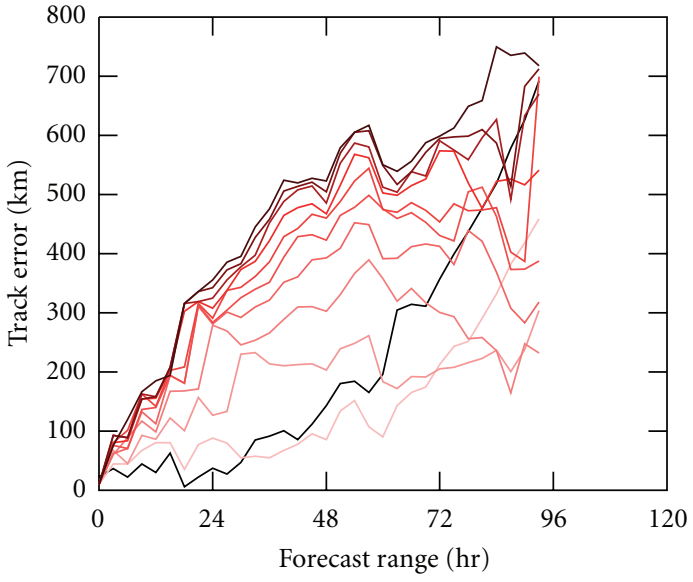

(b)

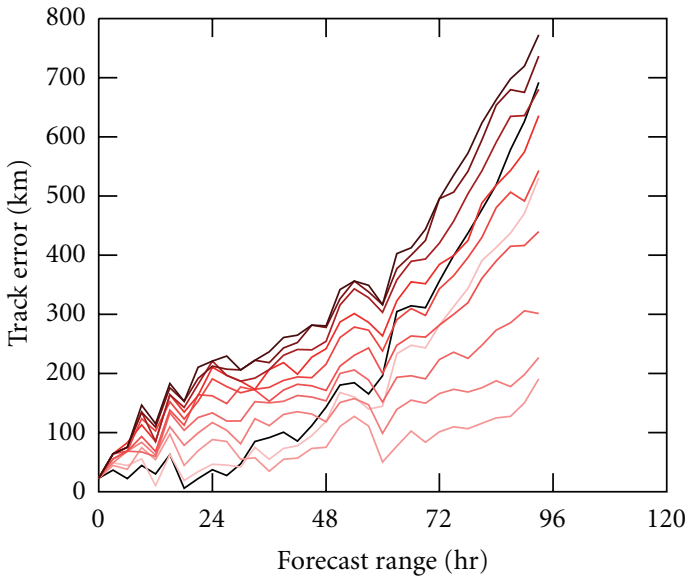

(d)

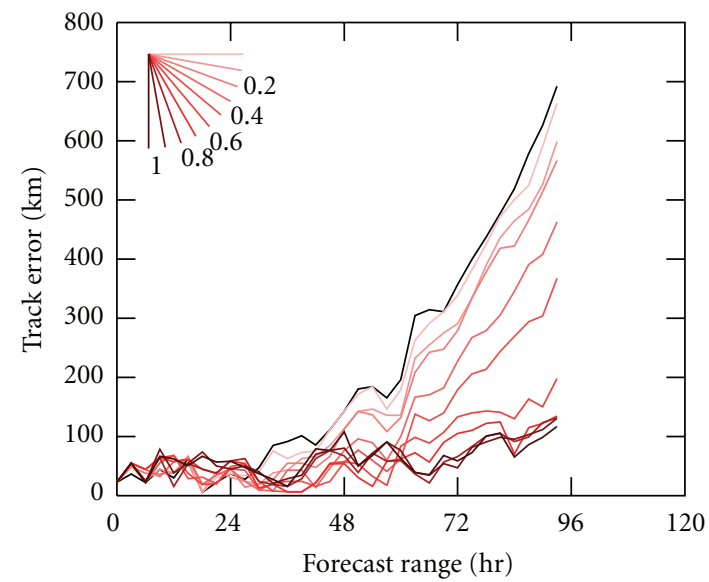

(f)

Figure 3: The predicted typhoon tracks for the runs initialized at 1800 UTC, 11 September 2008. The left column shows the tracks and the right column shows the forecast error defined by the distance between the predicted and observed (best) track. (a) and (b): the 10 cases with LEN_SCALING $=1.0$ and VAR_SCALING $=0.1-1.0$ step 0.1 . The color scale for the 10 cases with increasing VAR_SCALING is shown at the upper left corner of panel (f). The black curve is the control run with no AIRS data and the blue curve is the observed best track. (c) and (d): similar to (a) and (b) but for the 10 runs with LEN_SCALING $=0.5$ and VAR_SCALING $=0.1-1.0$ step 0.1. (e) and (f): same as (a) and (b) but for the 10 runs with LEN_SCALING $=0.1$ and VAR_SCALING $=0.1-1.0$ step 0.1 . 


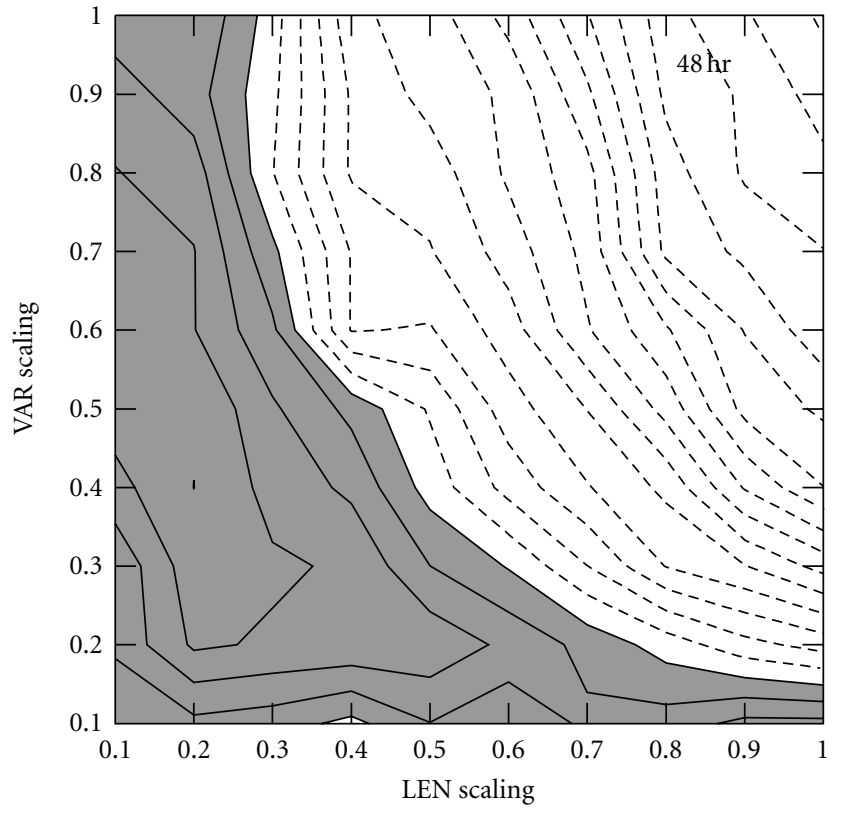

(a)

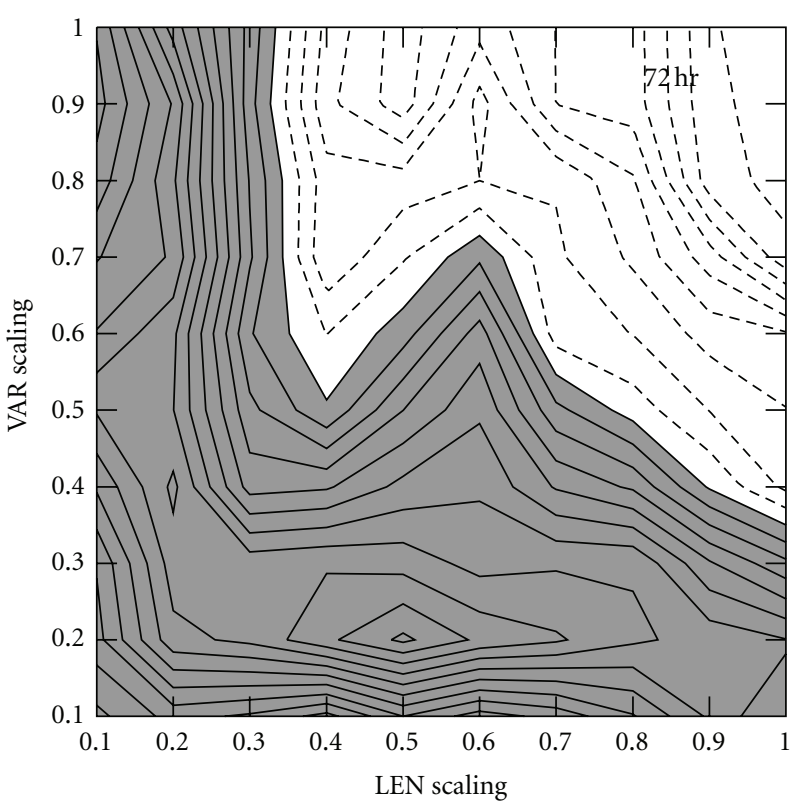

(b)

FIGURE 4: The contour plots for the "forecast error of the control run" minus "forecast error of an individual forecast with AIRS data" under a given pair of values of LEN_SCALING (abscissa) and VAR_SCALING (ordinate). The forecast error is for the typhoon track measured by the distance between the predicted and observed (best track) locations of the center of the typhoon. A positive value in the plot indicates that the forecast with AIRS data produces a smaller error compared to the control run (that does not incorporate AIRS data). Contour interval is $25 \mathrm{~km}$. Positive values are shaded in gray.

have a substantially higher horizontal resolution than conventional sounding. Since many small-scale structures in the AIRS data are real instead of bogus, it is beneficial to retain them, instead of suppressing them by using a larger value of LEN_SCALING as one would do for assimilating conventional soundings.

When the assimilation of the AIRS data leads to an improved forecast of typhoon tracks, the improvement usually becomes clear only after more than one day of forecast time. This likely reflects the fact that the AIRS data first affects the outflow regions (see the colored area in Figure 1 where the AIRS data passed quality control) before its influence reaches the center of the typhoon. Choosing two representative forecast times of 48 and 72 hours, Figure 4 shows the relative error in the typhoon track from a more comprehensive set of parameter-sweeping experiments with 100 pairs of LEN_SCALING (abscissa) and VAR_SCALING (ordinate), each parameter varied from 0.1 to 1.0 step 0.1 . The forecasts are initialized at 1800 UTC, 11 September 2008 (same as Figure 3). The contour plots are for the "forecast error of control run (no AIRS data)" minus "forecast error of a forecast with AIRS data." A positive value, shaded in gray in Figure 4, indicates that a forecast with AIRS data produces a smaller error than the control run, that is, the satellite data has a positive impact on the forecast. Figure 4 reaffirms that smaller values of one or both of the parameters are more desirable for the forecast of track in our case. To help visualize the predicted tracks corresponding to the contour plots, selected tracks from the runs with VAR_SCALING $=$ 0.1 and LEN_SCALING $=0.5-1.0$ are shown in Figure 5 .

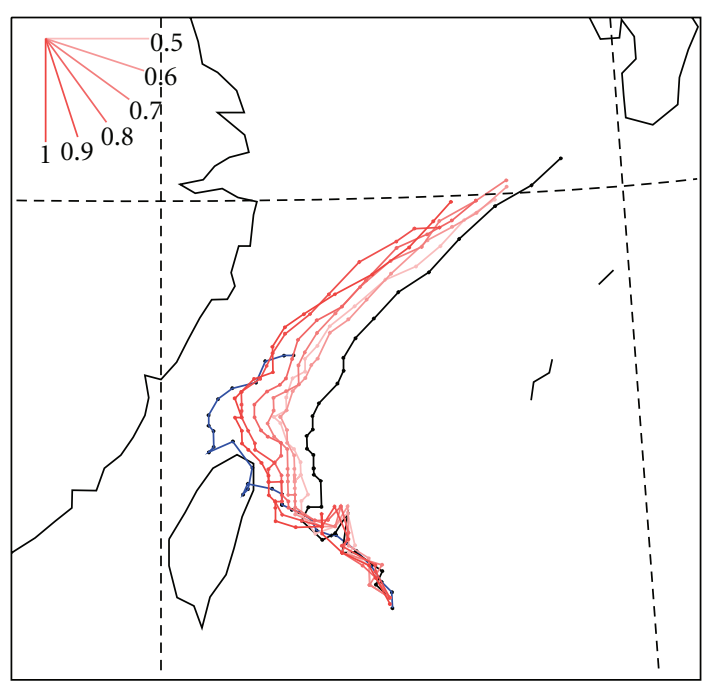

FIgURE 5: The tracks from forecasts initialized at 1800 UTC, 12 September 2008, with VAR_SCALING $=0.1$ and LEN_SCALING $=$ $0.5-1.0$ step 0.1 . Color scale is shown at the upper left corner, black curve is the control run without AIRS data, and blue curve is the observed track.

We have performed another set of forecast runs that are initialized at a later time of 1800 UTC, 12 September 2008. (In this case, the initial field is from a 6-hr forecast.) At that time, the center of typhoon Sinlaku is already close to the island of Taiwan. Figure 6 shows the predicted tracks for this case with LEN_SCALING fixed at 1.0 and VAR_SCALING 


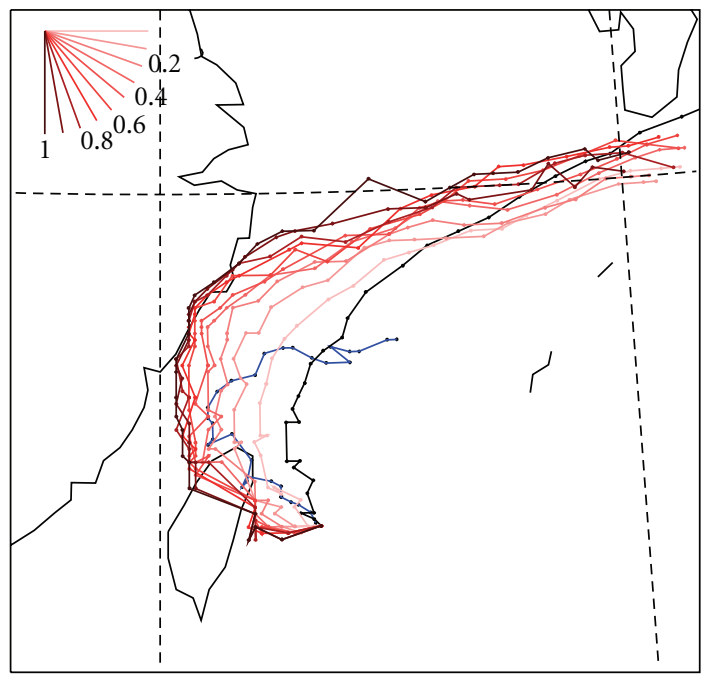

FIgURE 6: The forecasts initialized at 18 UTC, 12 September 2008, with LEN_SCALING $=1.0$ and VAR_SCALING $=0.1-1.0$ step 0.1 . Color scale is shown at the upper left corner, black curve is the control run without AIRS data, and blue curve is the observed track.

varied from $0.1-1.0$ step 0.1 . The qualitative behavior of the forecast is similar to the case initialized on 11 September 2008 as shown in Figure 3(a). The impact of adding the AIRS observation is to shift the predicted track westward. Again, with a large value of VAR_SCALING, the track is shifted too far west, producing a forecast error greater than the control run. The cases with smaller values of VAR_SCALING produced slightly improved forecast. Similar to the experiments shown in Figure 3, when we decrease LEN_SCALING, the overall modification of the forecast induced by the satellite data diminishes; since the modification in Figure 6 is already smaller than that in Figure 3(a), the counterparts of Figures 3(c) and 3(d) for this case produced even smaller modification of the forecast (not shown).

Overall, the improvement of the prediction for typhoon tracks is clearer with a reduction of LEN_SCALING away from the default, while the dependence of the forecast error on VAR_SCALING is slightly more mixed. Our results generally indicate that the default setting of the background error covariance in the WRF 3DVAR system (using the CV3 option) is far from optimal for assimilating the highresolution AIRS data for typhoon prediction. In addition to issues with spatial resolution discussed before, the unique pattern of summertime weather in the subtropics (the flow field is very intense along typhoon tracks but is scattered with random tropical convection elsewhere) might also affect the quality of the constructed background error. Although we could, in principle, reconstruct the background error by running extensive routine forecast using WRF at a higher resolution for the region of our interest, the background error so constructed will still be imperfect and require tuning. To this end, the generic framework of parametersweeping experiments explored in this study is potentially useful for practical applications. Our finding may also have implications for other applications of WRF in mesoscale

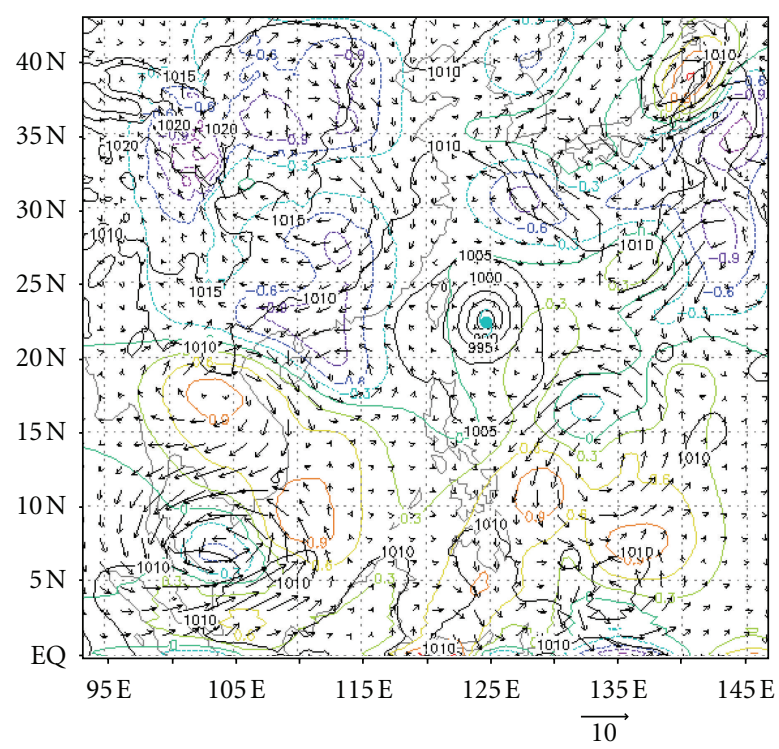

Figure 7: The modifications in the velocity and temperature fields in the initial condition due to assimilation of AIRS data. Shown is the case for the run initialized at 18 UTC, 11 September 2008, with LEN_SCALING $=0.5$ and VAR_SCALING $=0.2$. The vectors are the difference (defined as the outcome of a run with AIRS data minus that of the control run without AIRS) in the velocity at $500 \mathrm{hPa}$. Color contours are the difference in temperature, and black contours are the sea level pressure of the control run. The blue filled circle indicates the center of typhoon.

predictions unrelated to typhoon. We caution against blindly adopting the default background error when assimilating high-resolution (nonconventional) observations to WRF.

We also note that the modification of the flow field due to the assimilation of AIRS data is generally complicated. The modification in the initial temperature and velocity fields induced by the AIRS data usually permeates the entire model domain (see an example in Figure 7), even if the AIRS profiles near the center of the typhoon are not used (see Figure 1). Thus, the modification in the predicted typhoon tracks shown in this study was due as much to the modification of the outflow as it is due to the modification in the structure of the typhoon in the vicinity of its core.

Our parameter sweeping experiments have focused on the sensitivity of the forecast with respect to a perturbation of the background error matrix $\mathbf{B}$. In general (as a reviewer pointed out), one may also perturb the observation error matrix E. This will require the exploration of a much larger parameter space and is beyond the scope of our study (but can be a useful direction of future work). The purpose of this work was to explore numerical sensitivity rather than to pick out an "optimal" setting of the parameters, the latter may generally depend on the large-scale basic state of the individual cases of typhoons/hurricanes. Nevertheless, the range of sensitivity demonstrated in this study is itself useful as it quantifies the impact of tuning the background error when assimilating satellite data for the forecast. 


\section{Concluding Remarks}

Our results show the potential of improving the prediction of typhoon tracks by assimilating the AIRS observation. At the same time, we find that appropriate tuning of the background error in the data assimilation procedure is essential for the AIRS data to have a positive impact on the forecast. For the case of typhoon Sinlaku, the simulations made with small or moderate (still smaller than WRF's default) values of LEN_SCALING produced improved forecast. The dependence of the forecast error on VAR_SCALING is more mixed but the "best" value to adopt in our case is generally smaller than the default value. We must reiterate that the "optimal" values of these key parameters may vary from case to case. It will be beneficial to extend our study to a larger number of typhoons with different large-scale basic states, when sufficient AIRS (or other types of satellite) observations become available in the future. The problem may become even more complicated if we attempt to assimilate multiple types of satellite observations or a hybrid of satellite and conventional observations. Nevertheless, our results present a clear case that tuning the background error is critical if one wishes to make the best uses of AIRS observation in the forecast. Without carefully dealing with this aspect, errors can occur in the data assimilation process even if the satellite observation itself is of high quality and high density in space. Our study provides a framework for future systematic investigations on this issue.

\section{Acknowledgments}

The authors thank anonymous reviewers for useful comments that helped improve the manuscript. They are also grateful to Edward Olsen and Joel Susskind from NASA AIRS Team for useful communications. The first author thanks Dr. Shi-Shi Chi and Mrs. Wen-May Chen of Central Weather Bureau in Taiwan for help in plotting the results. The second author acknowledges support by NSF AGS-0934592.

\section{References}

[1] A. P. McNally, P. D. Watts, J. A. Smith et al., "The assimilation of AIRS radiance data at ECMWF," Quarterly Journal of the Royal Meteorological Society, vol. 132, no. 616, pp. 935-957, 2006.

[2] M. T. Chahine, T. S. Pagano, H. H. Aumann et al., "Improving weather forecasting and providing new data on greenhouse gases," Bulletin of the American Meteorological Society, vol. 87, no. 7, pp. 911-926, 2006.

[3] H. H. Aumann, M. T. Chahine, C. Gautier et al., "AIRS/AMSU/ HSB on the aqua mission: design, science objectives, data products, and processing systems," IEEE Transactions on Geoscience and Remote Sensing, vol. 41, no. 2, pp. 253-264, 2003.

[4] S.-H. Chou, B. Zavodsky, G. J. Jedlovec, and W. Lapenta, "Assimilation of atmospheric infrared sounder (AIRS) data in a regional model," Proceedings of the 14th Conference on Satellite Meteorology and Oceanography, 2006.

[5] J. Li and H. Liu, "Improved hurricane track and intensity forecast using single field-of-view advanced IR sounding measurements," Geophysical Research Letters, vol. 36, no. 11, Article ID L11813, 2009.

[6] H. Liu and J. Li, "An improvement in forecasting rapid intensification of Typhoon Sinlaku (2008) using clear-sky full spatial resolution advanced IR soundings," Journal of Applied Meteorology and Climatology, vol. 49, no. 4, pp. 821-827, 2010.

[7] J. Li, H. Liu, and T. J. Schmit, "Advanced IR measurements improve hurricane forecasts," in Remote Sensing, Proceedings of the SPIE Newsletter, 2010.

[8] D. M. Barker, W. Huang, Y. R. Guo, A. J. Bourgeois, and Q. N. Xiao, "A three-dimensional variational data assimilation system for MM5: implementation and initial results," Monthly Weather Review, vol. 132, no. 4, pp. 897-914, 2004.

[9] T. Zhu, D. L. Zhang, and F. Weng, "Impact of the advanced microwave sounding unit measurements on hurricane prediction," Monthly Weather Review, vol. 130, no. 10, pp. 24162432, 2002.

[10] X. Zhang, Q. Xiao, and P. J. Fitzpatrick, "The impact of multisatellite data on the initialization and simulation of Hurricane Lili's (2002) rapid weakening phase," Monthly Weather Review, vol. 135, no. 2, pp. 526-548, 2007.

[11] C. B. Chou, C. Y. Huang, H. P. Huang, K. H. Wang, and T. C. Yeh, "The analysis of typhoon structures using advanced microwave sounding unit data and its application to prediction," Journal of Applied Meteorology and Climatology, vol. 47, no. 5, pp. 1476-1492, 2008.

[12] J. S. Goerss, "Impact of satellite observations on the tropical cyclone track forecasts of the navy operational global atmospheric prediction system," Monthly Weather Review, vol. 137, no. 1, pp. 41-50, 2009.

[13] W. C. Skamarock, J. B. Klemp, J. Dudhia et al., "A description of the advanced research WRF Version 3," NCAR Technical Note, 2008.

[14] WRF-ARW, Weather Research and Forecasting ARW Version 3 Modeling System User's Guide, National Center for Atmospheric Research, 2009.

[15] A. C. Lorenc, "Analysis methods for numerical weather prediction," Quarterly Journal of the Royal Meteorological Society, vol. 112, no. 474, pp. 1177-1194, 1986.

[16] D. F. Parrish and J. C. Derber, "The National Meteorological Center's spectral statistical- interpolation analysis system," Monthly Weather Review, vol. 120, no. 8, pp. 1747-1763, 1992.

[17] G. Desroziers and S. Ivanov, "Diagnosis and adaptive tuning of information error parameters in a variational assimilation," Quarterly Journal of the Royal Meteorological Society, vol. 127, pp. 1433-1452, 2001.

[18] W. Sadiki and C. Fischer, "A posteriori validation applied to the 3D-VAR Arpège and Aladin data assimilation systems," Tellus, vol. 57, no. 1, pp. 21-34, 2005.

[19] J. Susskind, C. D. Barnet, and J. M. Blaisdell, "Retrieval of atmospheric and surface parameters from AIRS/AMSU/HSB data in the presence of clouds," IEEE Transactions on Geoscience and Remote Sensing, vol. 41, no. 2, pp. 390-409, 2003.

[20] J. Susskind, C. D. Barnet, J. Blaisdell et al., "Accuracy of geophysical parameters derived from Atmospheric Infrared Sounder/Advanced Microwave Sounding Unit as a function of fractional cloud cover," Journal of Geophysical Research D, vol. 111, no. 9, Article ID D09S17, 19 pages, 2006.

[21] J. Susskind, J. M. Blaisdell, L. Iredell, and F. Keita, "Improved temperature and quality control methodology using AIRS/ AMSU data: the AIRS Science team version 5 retrieval algorithm," IEEE Transactions on Geoscience and Remote Sensing, vol. 49, pp. 833-907, 2011. 
[22] E. T. Olsen and Contributors, AIRS/AMSU/HSB Version 5 data release user guide, Jet Propulsion Laboratory, pp. 68, 2007, http://disc.sci.gsfc.nasa.gov/AIRS/documentation.

[23] Z. Q. Liu and F. Rabier, "The potential of high-density observations for numerical weather prediction: a study with simulated observations," Quarterly Journal of the Royal Meteorological Society, vol. 129, no. 594, pp. 3013-3035, 2003.

[24] P. Bauer, R. Buizza, C. Cardinali, and J. Noël Thépaut, "Impact of singular-vector-based satellite data thinning on NWP," Quarterly Journal of the Royal Meteorological Society, vol. 137, no. 655 , pp. 286-302, 2011.

[25] N. Bormann and P. Bauer, "Estimates of spatial and interchannel observation-error characteristics for current sounder radiances for numerical weather prediction. I: methods and application to ATOVS data," Quarterly Journal of the Royal Meteorological Society, vol. 136, no. 649, pp. 1036-1050, 2010.

[26] N. Bormann, A. Collard, and P. Bauer, "Estimates of spatial and interchannel observation-error characteristics for current sounder radiances for numerical weather prediction. II: application to AIRS and IASI data," Quarterly Journal of the Royal Meteorological Society, vol. 136, no. 649, pp. 1051-1063, 2010.

[27] J. Gu, Q. Xiao, Y. H. Kuo, D. M. Barker, J. Xue, and X. Ma, "Assimilation and simulation of typhoon Rusa (2002) using the WRF system," Advances in Atmospheric Sciences, vol. 22, no. 3, pp. 415-427, 2005.

[28] Y.-R. Guo, H.-C. Lin, X. X. Ma, X.-Y. Huang, C. T. Terng, and Y.-H. Kuo, "Impact of WRF-Var (3dVar) background error statistics on typhoon analysis and forecast," in Proceedings of the 7th Annual WRF Users' Workshop, Boulder, Colo, USA, June 2006.

[29] S. R. H. Rizvi, WRF-Var Tutorial, WRFDA: Background error estimation, 2009, http://box.mmm.ucar.edu/wrf/users/wrfda/ Tutorials/2009_July. 

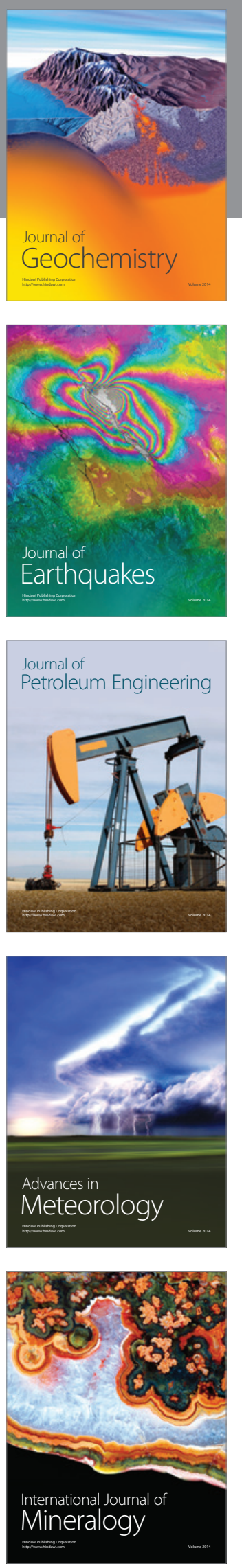
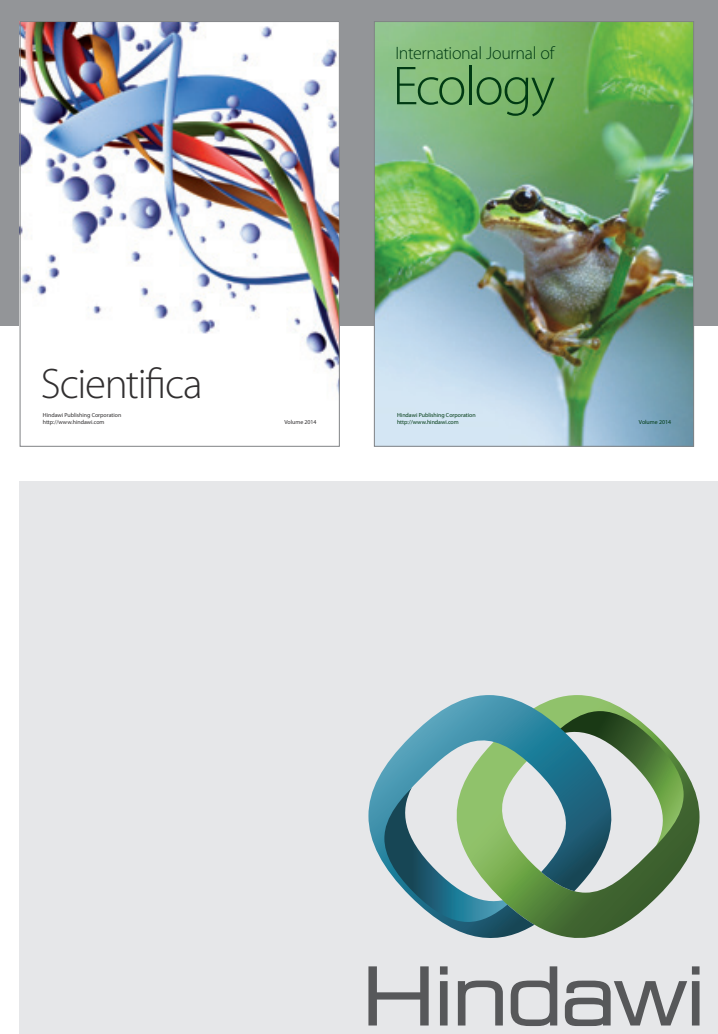

Submit your manuscripts at http://www.hindawi.com
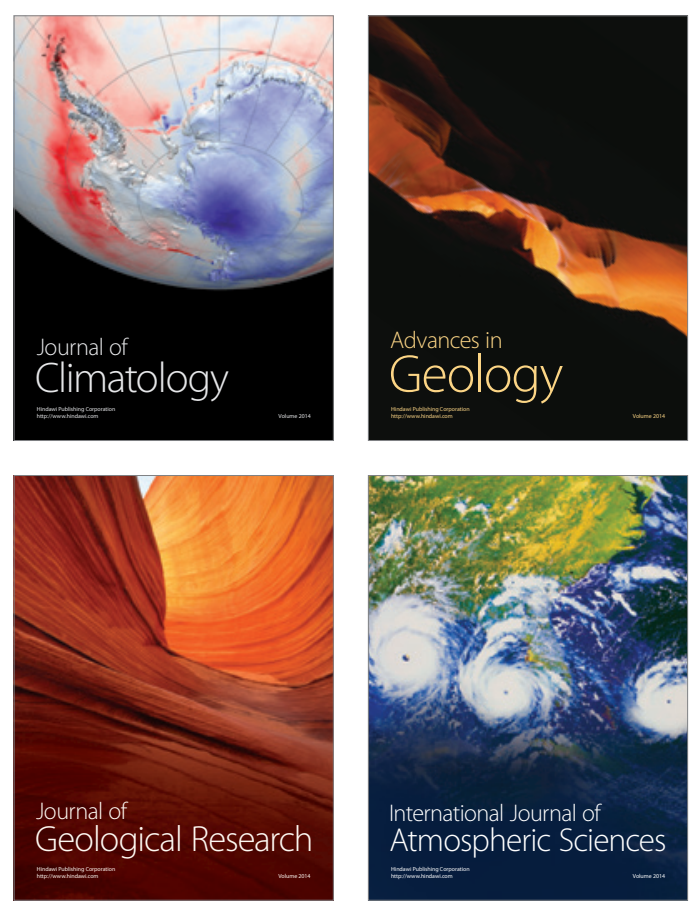
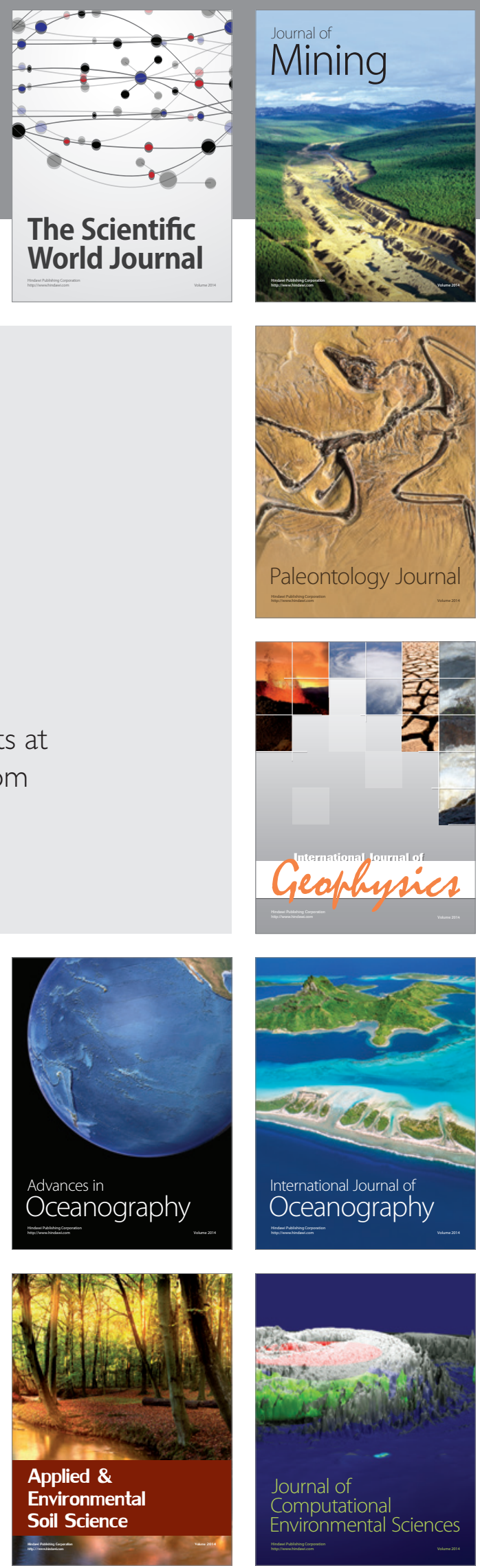